\title{
GENERALIZED HISTOGRAM EQUALIZATION BASED ON LOCAL CHARACTERISTICS
}

\author{
Tzu-Cheng Jen and Sheng-Jyh Wang \\ Inst. of Electronics, National Chiao Tung University, Hsinchu, Taiwan, R.O.C. \\ E-mail: shengjyh@cc.nctu.edu.tw
}

\begin{abstract}
Histogram Equalization (HE) and its variations have been widely used in image enhancement. Even though these approaches may enhance image contrast in an effective and efficient way, they usually face some undesired drawbacks, like loss of image details, noise amplification and overenhancement. In this paper, we propose a generalized histogram equalization technique based on localized image analysis. Starting from designing two measures $\mathrm{f}_{1}$ and $\mathrm{f}_{2}$ to measure local characteristics around each pixel, the global statistics of these two local measures are then recorded into an extended histogram. Based on this extended histogram, we develop a procedure to generate suitable intensity transfer functions for various applications, like contrast enhancement and shadow enhancement. Experimental results show that the proposed algorithm provides a flexible and efficient way for image enhancement.
\end{abstract}

Index Terms - Image enhancement

\section{INTRODUCTION}

Histogram Equalization (HE) has been widely used for image enhancement [1]. In HE, the cumulative density function (cdf) of the histogram is used as the intensity transfer function for intensity value mapping. This approach enhances the contrast of an image by expanding dynamic range of the original image to all available dynamic range. Since the HE approach considers only global statistics of the image, some image details may get lost while some portions of the image may get over-enhanced. Moreover, image noise may also get enhanced during the contrast enhancement process.

To overcome the over-enhancement problem, [2] and [3] proposed similar solutions that preserve the intensity mean of the image data by using sub-histogram information. However, under some circumstances, the improvement of the image quality may be restricted due to the meanpreserving criterion. On the other hand, [4] and [5] performed contrast enhancement based on adaptive histogram equalization (AHE). The operation of AHE is basically the same as HE, except that the histogram is now formed from localized data. However, computational complexity can be a major concern and the enhanced results may look unnatural.

In this paper, we propose an extension of histogram equalization for image enhancement. By taking local characteristics into account, a more general approach is developed. This approach can be applied to various image enhancement applications, like contrast enhancement and shadow enhancement. In this paper, the concept of the proposed approach is first introduced in Section 2. Its applications to contrast enhancement and shadow enhancement are then demonstrated in Section 3. Finally, conclusions are given in Section 4.

\section{GENERALIZED HISTOGRAM EQUALIZATION}

2.1 Generation of Histogram in $\mathrm{HE}$

In histogram equalization, the first step is to generate the histogram of the image. Then the intensity transfer function for contrast enhancement is obtained as

$$
T(g)=g_{\text {min }}+\left(g_{\text {max }}-g_{\text {min }}\right) \cdot \frac{\int_{g_{\text {min }}}^{g} h(x) d x}{\int_{g_{\text {min }}}^{g_{\max }} h(x) d x}
$$

where $g$ denotes the intensity value, $g_{\min }$ and $g_{\max }$ denote the lower bound and upper bound of $g, h(x)$ denotes the histogram of the image, and $T(\mathrm{~g})$ denotes the intensity transfer function for contrast enhancement. Intuitively, a large peak in the histogram causes a steep increase in the cdf function. Hence, this approach allocates more gray levels for frequent intensity values, while assigns less gray levels for infrequent intensity values.

Actually, this histogram equalization process can also be explained from a different viewpoint. Here, we may imagine $\mathrm{h}(\mathrm{x})$ as an expansion function, which describes how likely the intensity value $x$ needs to be expanded for image enhancement. In $\mathrm{HE}$, if an intensity value $\mathrm{X}$ occurs more frequently, then we tend to expand more these intensity values around X. Hence, Equation (1) can also be explained as the reallocation of intensity values based on the distribution of an expansion function $h(x)$.

On the other hand, the generation of the histogram $h(x)$ can be viewed as a masking-and-accumulating operation. Imagine we use a $1 \times 1$ mask to scan through the image to measure the intensity data at each pixel. These intensity 
values are then accumulatively added into $h(x)$. By repeating the masking-and-accumulating operation over the whole image, the histogram function $\mathrm{h}(\mathrm{x})$ is obtained. In Figure 1, we illustrate such a masking-and-accumulating operation. Due to the limited size of the $1 \times 1$ mask, the $\mathrm{HE}$ method doesn't consider the neighboring information around each pixel. Hence, the histogram function $\mathrm{h}(\mathrm{x})$ contains only global statistics of the image.

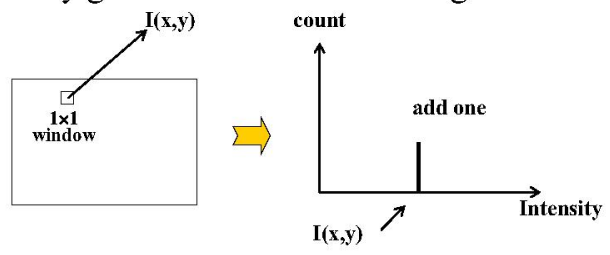

Figure 1 Illustration of histogram generation

\subsection{Proposed Algorithm}

\subsubsection{Extended Histogram}

In our approach, we first extend the scanning mask size from $1 \times 1$ to $n \times n$. When the center of the mask is placed at $(x, y)$, we define $\mathrm{I}(\mathrm{x}, \mathrm{y})$ as the intensity value at the center pixel, while define $\mathrm{N}(\mathrm{x}, \mathrm{y})$ as the set of intensity values within the $n \times n$ neighborhood of $(x, y)$. The definitions of $\mathrm{I}(\mathrm{x}, \mathrm{y})$ and $\mathrm{N}(\mathrm{x}, \mathrm{y})$ are illustrated in Figure 2(a). Besides, we propose the use of two measures $f_{1}(I(x, y), N(x, y))$ and $\mathrm{f}_{2}(\mathrm{I}(\mathrm{x}, \mathrm{y}), \mathrm{N}(\mathrm{x}, \mathrm{y}))$ to estimate some local characteristics within the mask window. Here, we define $f_{1}$ and $f_{2}$ to measure the local average and local variations within the mask, respectively. For example, if we choose the mask size to be $3 \times 3$, we may define $f_{1}$ and $f_{2}$ to be

$$
\begin{array}{r}
f_{1}(x, y)=\frac{1}{9} \sum_{i=-1}^{1} \sum_{j=-1}^{1} I(x+i, y+j) \\
f_{2}(x)=\operatorname{Max}\{I(x+i, y+j) ;-1 \leq i \leq 1,-1 \leq j \leq 1\} \\
-\operatorname{Min}\{I(x+i, y+j) ;-1 \leq i \leq 1,-1 \leq j \leq 1\}
\end{array}
$$

In fact, many other functions, like a weighted mean and the local variance, can also be used to define $f_{1}$ and $f_{2}$.

With the definitions of $\mathbf{f}_{1}$ and $\mathbf{f}_{2}$, we can calculate $\mathrm{f}_{1}(\mathrm{I}(\mathrm{x}, \mathrm{y}), \mathrm{N}(\mathrm{x}, \mathrm{y}))$ and $\mathrm{f}_{2}(\mathrm{I}(\mathrm{x}, \mathrm{y}), \mathrm{N}(\mathrm{x}, \mathrm{y}))$ at each pixel $(\mathrm{x}, \mathrm{y})$. As the mask scans through the image, we count the occurrence frequency of $\left(f_{1}, f_{2}\right)$ and generate a so-called "extended histogram". Figure 2(b) illustrates the generation of this extended histogram. This operation is similar to the operation illustrated in Figure 1, except that the mask size is now $n \times n$ and the intensity value $I(x, y)$ is replaced by two local measures $\mathbf{f}_{1}$ and $\mathbf{f}_{2}$

After the generation of the extended histogram, we perform normalization over the extended histogram to get the 2-D probability density function $\mathbf{p}\left(\mathbf{f}_{\mathbf{1}}, \mathbf{f}_{2}\right)$. In theory, $\mathrm{p}\left(\mathbf{f}_{1}, \mathbf{f}_{2}\right)$ records the global statistics of the local characteristics. For example, if $\mathrm{p}(\alpha, \beta)=\mathrm{k}$, it means there exists some pixels in the image with local features being $\mathbf{f}_{1}=\alpha$ and $\mathbf{f}_{2}=\beta$. Moreover, the occurrence rate of these pixels is $\mathrm{k}$.

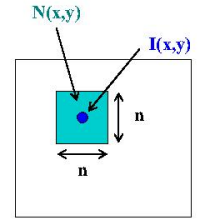

(a)

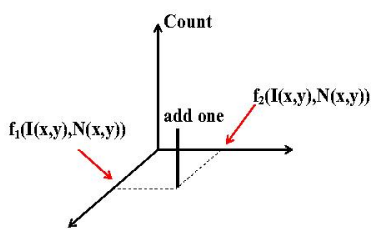

(b)
Figure 2 (a) Illustration of $\mathrm{I}(\mathrm{x}, \mathrm{y})$ and $\mathrm{N}(\mathrm{x}, \mathrm{y})$.

(b)Generation of the extended histogram

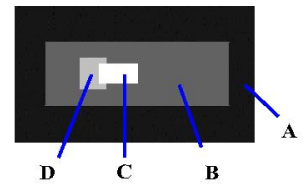

(a)

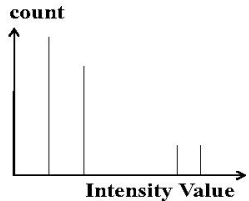

(b)

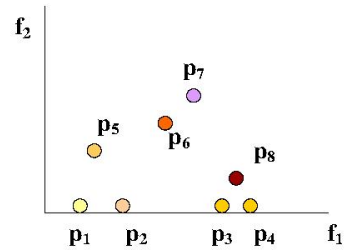

(c)

Figure 3 (a) Synthesized image.

(b) Histogram.

(c) $p\left(f_{1}, f_{2}\right)$ of (a).

In Figure 3(a), we show a synthesized image, which contains 4 smooth regions, A, B, C, and D. The histogram of this image is shown in Figure 3(b). Due to the fact that $C$ and D occupy only a small portion of the image, it is expected that the contrast between $\mathrm{C}$ and $\mathrm{D}$ cannot be adequately enhanced by the HE algorithm. In Figure 3(c), we show an illustration of the 2-D pdf function $\mathrm{p}\left(\mathrm{f}_{1}, \mathrm{f}_{2}\right)$ of Figure 3(a). Here, $p_{1}, p_{2}, p_{3}$, and $p_{4}$ correspond to the smooth parts of $\mathrm{A}, \mathrm{B}, \mathrm{C}$, and $\mathrm{D}$, respectively. On the other hand, $\mathrm{p}_{5}, \mathrm{p}_{6}, \mathrm{p}_{7}$ and $\mathrm{p}_{8}$ correspond to the boundary regions between $\mathrm{A}$ and $\mathrm{B} ; \mathrm{B}$ and $\mathrm{C} ; \mathrm{B}$ and $\mathrm{D}$; and $\mathrm{C}$ and $\mathrm{D}$. Here, we use a brighter color to represent a larger value of $p\left(f_{1}, f_{2}\right)$ while use a darker color to represent a smaller value. It can be easily seen that this 2-D pdf function $p\left(f_{1}, f_{2}\right)$ offers much more useful information than the 1-D histogram. It records not only the distribution of intensity values but also the distribution of local variations. Based on $\mathrm{p}\left(\mathrm{f}_{1}, \mathrm{f}_{2}\right)$, we can easily distinguish pixels in smooth regions from pixels at boundary regions. This capability enables more complicated manipulations for image enhancement.

\subsubsection{Expansion Function}

Based on $\mathrm{p}\left(\mathrm{f}_{1}, \mathrm{f}_{2}\right)$, we aim to develop a suitable intensity mapping function that can satisfactorily enhance image contents. In this paper, we propose the use of a "conditional expansion function" to generate the intensity transfer function. This conditional expansion function 
$S\left[g \mid\left(f_{1,} f_{2}\right)=(\alpha, \beta)\right]$ is defined as a function of intensity value $\mathrm{g}$, given $\mathrm{f}_{1}=\alpha$ and $\mathrm{f}_{2}=\beta$. That is, this function describes how likely we want the intensity values around $g$ to expand if we are given the condition that $\mathrm{f}_{1}=\alpha$ and $\mathrm{f}_{2}=\beta$. For example, if we want to enhance the image contrast in Figure 3(a) without enhancing image noise over smooth regions, we may simply set a threshold over $\mathrm{f}_{2}$ to screen out $\mathrm{p}_{1}, \mathrm{p}_{2}, \mathrm{p}_{3}$, and $\mathrm{p}_{4}$ first. On the other hand, for $\mathrm{p}_{5}, \mathrm{p}_{6}, \mathrm{p}_{7}$, and $\mathrm{p}_{8}$, which correspond to edge regions in the image, we may define the conditional expansion function to be something like

$$
S\left[g \mid\left(f_{1,} f_{2}\right)=(\alpha, \beta)\right] \equiv \Pi\left(\frac{g-\alpha}{\beta}\right),
$$

where $\Pi(x)$ is the rectangular function expressed as

$$
\Pi(x) \equiv\left\{\begin{array}{rr}
1 & -0.5 \leq \mathrm{x} \leq 0.5 \\
0 & \text { otherwise }
\end{array}\right.
$$

This means once we have observed a set of local statistics with $f_{1}=\alpha$ and $f_{2}=\beta$, then we expect the intensity values within the range $[\alpha-0.5 \beta, \alpha+0.5 \beta]$ are more likely to be expanded. Of course, there are many other choices of $\mathrm{S}\left[\mathrm{g} \mid \mathrm{f}_{1}, \mathrm{f}_{2}\right]$, depending on how we want the image to be enhanced. Moreover, since the value of $p\left(f_{1}, f_{2}\right)$ reflects the occurrence frequency of $\left(f_{1}, f_{2}\right)$, we may further take $p\left(f_{1}, f_{2}\right)$ into account and calculate the averaged expansion function:

$$
S(g)=\int_{f_{1, \text { min }}}^{f_{1, \max }} \int_{f_{2, \text { min }}}^{f_{2, \text { max }}} S\left[g \mid\left(f_{1}, f_{2}\right)\right] p\left(f_{1}, f_{2}\right) d f_{1} d f_{2} .
$$

This $\mathrm{S}(\mathrm{g})$ function indicates which intensity values are more likely to be stretched in the image enhancement process. Then, based on $\mathrm{S}(\mathrm{g})$, we can deduce the intensity transfer function $T(\mathrm{~g})$, which is defined as

$$
T(g)=g_{\min }+\left(g_{\max }-g_{\text {min }}\right) \cdot \frac{\int_{g_{\min }}^{g}[c+S(\tau)] d \tau}{\int_{g_{\min }}^{g_{\max }}[c+S(\tau)] d \tau}
$$

An illustration of $\mathrm{S}(\mathrm{g})$ and $\mathrm{T}(\mathrm{g})$ for the example of Figure $3(c)$ is shown in Figure 4. Here, we set a threshold over $f_{2}$ to ignore $\mathrm{p}_{1}, \mathrm{p}_{2}, \mathrm{p}_{3}$ and $\mathrm{p}_{4} . \mathrm{S}\left[\mathrm{g} \mid \mathrm{f}_{1}, \mathrm{f}_{2}\right]$ is defined as Equation (4) and Figure 4(a) shows the $\mathrm{S}\left[\mathrm{g} \mid \mathrm{f}_{1}, \mathrm{f}_{2}\right] \mathrm{p}\left(\mathrm{f}_{1}, \mathrm{f}_{2}\right)$ profiles corresponding to $\mathrm{p}_{5}, \mathrm{p}_{6}, \mathrm{p}_{7}$, and $\mathrm{p}_{8}$. Based on Equations (6) and $(7), c+S(g)$ and $\mathrm{T}(\mathrm{g})$ can be easily computed, as shown in Figure 4(b) and Figure 4(c).

It can be easily imagined that if $(\mathrm{c}+\mathrm{S}(\mathrm{g}))$ has a larger value at $\mathrm{g}$, then $\mathrm{T}(\mathrm{g})$ has a larger slope at $\mathrm{g}$. This means the intensity values around $g$ tend to be stretched more. Hence, after having obtained the expansion function $\mathrm{S}(\mathrm{g})$, we can deduce the intensity transfer function $\mathrm{T}(\mathrm{g})$ to assign intensity values based on the prominence of local statistics. Moreover, note that in Equation (7) we add one extra parameter $\mathrm{c}$. This parameter provides flexible control over the degree of image enhancement. A smaller value of $\mathrm{c}$ causes a more apparent adjustment, and vice versa. Moreover, it is worth mentioning that if we choose $S\left[g \mid f_{1}, f_{2}\right]$ $=\delta\left(g-f_{1}\right)$, then $S(g)$ degenerates to the normalized form of the 1-D histogram $\mathrm{h}(\mathrm{x})$.

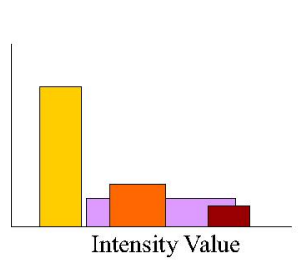

(a)

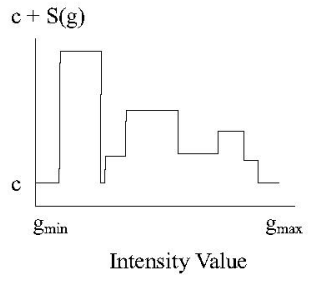

(b)

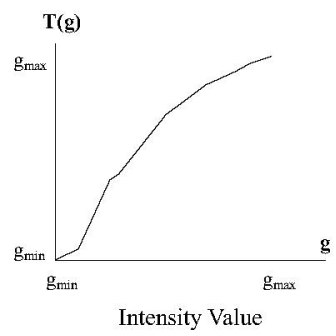

(c)

Figure 4 Example of $\mathrm{S}\left[\mathrm{g} \mid \mathrm{f}_{1}, \mathrm{f}_{2}\right] \mathrm{p}\left(\mathrm{f}_{1}, \mathrm{f}_{2}\right), \mathrm{c}+\mathrm{S}(\mathrm{g})$, and $\mathrm{T}(\mathrm{g})$.

\subsubsection{Prevention of Over-Enhancement}

As mentioned above, once we have gotten the expansion function $\mathrm{S}(\mathrm{g})$, we can generate the intensity transfer function based on Equation (7). In practice, the magnitude of $\mathrm{S}(\mathrm{g})$ has significant influence on the enhanced result. If there are some dominant peaks in S(g), overly enhanced images may be produced. To avoid this problem, we proposed the use of a magnitude mapping function $\mathrm{M}(\mathrm{)})$ to compress the dynamic range of $\mathrm{S}(\mathrm{g})$. This magnitude mapping function is a monotonically increasing function, with its slope monotonically decreasing to zero. An example of $M()$ is

$$
Y=M(X)=X^{1 / M_{0}}
$$

where $\mathrm{X}$ is the original expansion function, $\mathrm{Y}$ is the adjusted expansion function, and $\mathrm{M}_{0}$ is a control parameter. In summary, Equation (7) is further modified to be

$$
T(g)=g_{\min }+\left(g_{\max }-g_{\min }\right) \cdot \frac{\int_{g_{\min }}^{g}[c+M(S(\tau))] d \tau}{\int_{\max }^{\max }[c+M(S(\tau))] d \tau} .
$$

With the use of $\mathrm{M}(\mathrm{)})$, the expansion function with large magnitudes will be properly constrained to an extent so that the processed image looks more natural.

\subsection{Contrast Enhancement}

\section{APPLICATIONS}

In our simulations, an RGB-formated color image is first converted to the HSI color space and we apply our algorithm to the $I$ component only. Besides, $f_{1}$ and $f_{2}$ are defined as Equations (2) and (3). To perform contrast enhancement, we define $\mathrm{S}\left[\mathrm{g} \mid \mathrm{f}_{1}, \mathrm{f}_{2}\right]$ as 


$$
S\left[g \mid\left(f_{1,} f_{2}\right)=(\alpha, \beta)\right] \equiv\left\{\begin{array}{lr}
\Pi\left(\frac{g-\alpha}{\beta}\right) & \beta \geq 10 \\
0 & \text { otherwise }
\end{array}\right.
$$

In Figure 5, we show some simulations and the comparisons with HE, AHE and the method proposed by [3]. We can find that the proposed algorithm provides robust and more natural enhancement results. Besides, it is worth mentioning that the image quality in Figure $5(\mathrm{~d})$ is with little improvement due to the mean-preserving constraint used in [3], Moreover, even though there exists strong image noise in Figure 6(a), we may still properly enhance the image contrast without overly enhance the image noise.

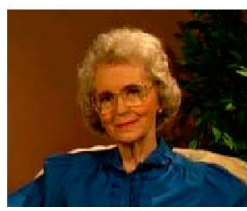

(a)

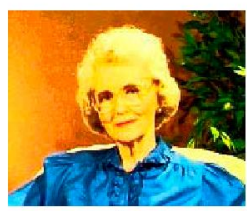

(b)

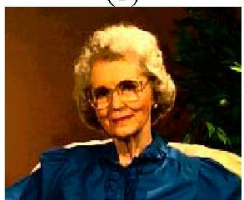

(d)

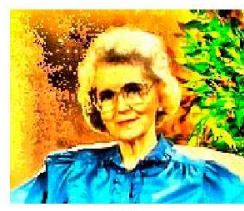

(c)

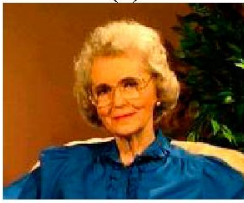

(e)
Figure 5. (a) Original image. (b) HE. (c) AHE. (d) Processed result of [3]. (e) Proposed method $(\mathrm{C}=0, \mathrm{Mo}=2)$.

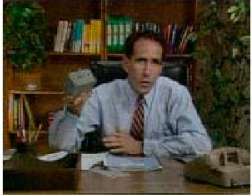

(a)

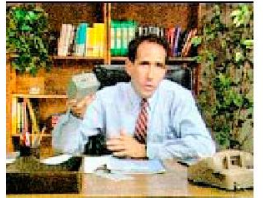

(b)

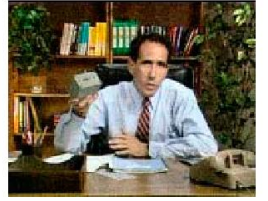

(c)
Figure 6. (a) Original image contaminates with Gaussian noise. (b) HE. (c) Proposed method (C=0, Mo=2).

\subsection{Shadow Enhancement}

The luminance variation within a scene often has a very wide dynamic range. Due to the narrower dynamic range of the capturing devices, some portions of the reproduced image may look too dark or too bright. In this section, we demonstrate how the proposed method can be used to deal with the shadow enhancement problem.

For shadow enhancement, we may enhance areas with smaller gradients while suppress areas with larger gradients. This concept was proposed by [6] for dynamic range compression. To achieve the same goal, here we may redefine the conditional expansion function to be

$$
S\left[g \mid\left(f_{1,} f_{2}\right)=(\alpha, \beta)\right] \equiv k(\beta) \Pi\left(\frac{g-\alpha}{\beta}\right)
$$

where $\mathrm{k}(\beta)$ is large for small $\beta$, and vice versa. To suppress image noise, we may also set $k(\beta)$ to be zero if $\beta$ is smaller than a pre-determined threshold. Some experimental results of shadow enhancement are shown in Figure 7. It can be seen that dark regions are effectively enhanced.

\section{CONCLUSIONS}

In this paper, we propose a new approach for image enhancement. In this approach, we extend the concept of histogram equalization to record the global statistics of some local characteristics. A complete procedure for the generation of the intensity transfer function is developed. Experimental results demonstrate that the proposed approach can provide a reliable and flexible scheme for various image enhancement applications.

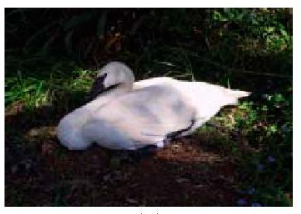

(a)

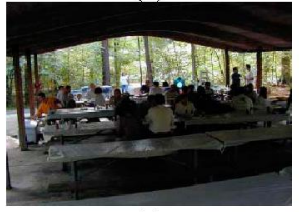

(c)

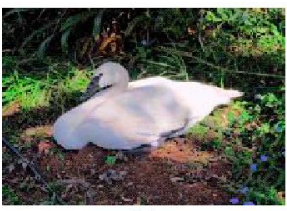

(b)

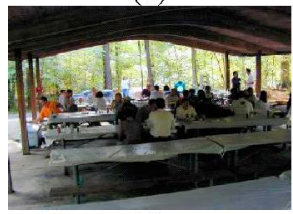

(d)
Figure 7. (a) (c): Original images.(b)(d): Images enhanced by the proposed method. $\left(\mathrm{c}=0, \mathrm{M}_{\mathrm{o}}=2\right)$

\section{ACKNOWLEDGEMENT}

This research was supported by National Science Council of the Republic of China under Grant Number NSC-93-2219E-009-017.

\section{REFERENCES}

[1] R.C. Gonzalez and R. E. Woods, Digital image processing, Addison-Wesley, 2002.

[2] Y.T. Kim, "Contrast enhancement using brightness- preserving bi-histogram equalization," IEEE Trans. on Consumer Electronics, Vol. 43, No. 1, pp.1-8, Feb. 1997.

[3] Soong-Der Chen, Abd. Rahman Ramli, "Minimum mean brightness error Bi-histogram equalization in contrast enhancement," IEEE Trans. on Consumer Electronics, Vol. 49, No. 4, pp.1310-1319, Nov. 2003.

[4] J.Y. Kim, L.S. Kim and S.H. Hwang, "An advanced contrast enhancement using partially overlapped sub-block histogram equalization," IEEE Trans. on Circuits and Systems for Video Technology, Vol. 11, Issue 4, pp.475-484, Apr. 2001

[5] T.K. Kim, J.K. Paik and B.S. Kang, "Contrast enhancement system using spatially adaptive histogram equalization with temporal filtering," IEEE Trans. on Consumer Electronics, Vol. 44, Issue 1, pp.82-87, Feb. 1998.

[6]R. Fattal, D. Lischinski and M. Werman, "Gradient domain high dynamic range compression", Proc. ACM SIGGRAPH'02 